Special issue in honor of Prof. George C. Papageorgiou

\title{
Differences in susceptibility to photoinhibition do not determine growth rate under moderate light in batch or turbidostat - a study with five green algae
}

\author{
H. MATTILA* (iD) D. VALEV*, K.B. MISHRA**(D), V. HAVURINNE* (D) O. VIRTANEN* (D), \\ M. ANTINLUOMA*, and E. TYYSTJÄRVI*, \\ Department of Life Technologies/Molecular Plant Biology, University of Turku, 20014 Turku, Finland \\ Global Change Research Institute of the Czech Academy of Sciences, Bèlidla 986, 4a, 60300 Brno, Czech \\ Republic**
}

\begin{abstract}
To understand growth limitations of photosynthetic microorganisms, and to investigate whether batch growth or certain photosynthesis-related parameters predict a turbidostat (continuous growth at constant biomass concentration) growth rate, five green algal species were grown in a photobioreactor in batch and turbidostat conditions and their susceptibilities to photoinhibition of photosystem II as well as several photosynthetic parameters were measured. Growth rates during batch and turbidostat modes varied independently of each other; thus, a growth rate measured in a batch cannot be used to determine the continuous growth rate. Greatly different photoinhibition susceptibilities in tested algae suggest that different amounts of energy were invested in repair. However, photoinhibition tolerance did not necessarily lead to a fast growth rate at a moderate light intensity. Nevertheless, we report an inverse relationship between photoinhibition tolerance and minimum saturating irradiance, suggesting that fast electron transfer capacity of PSII comes with the price of reduced photoinhibition tolerance.
\end{abstract}

Keywords: Chlorococcum novae-angliae; Desmodesmus quadricauda; Ettlia oleoabundans; microalga; photodamage; photoinactivation; rapid light curve; Scenedesmus ecornis; Scenedesmus obliquus.

\section{Introduction}

The potential of microalgae or cyanobacteria to produce food, biomass, fuel, various high-value compounds, and electricity or to clean wastewater is intensively studied (for reviews, see Borowitzka and Vonshak 2017, Stensjö et al. 2018). To obtain a commercially viable yield of the desired product and to keep contamination in control, these

\section{Highlights}

- Growth rate of a dilute batch does not predict growth rate in a dense turbidostat

- Susceptibility to PSII photoinhibition does not necessarily limit growth

- Fast forward electron transfer of PSII may lead to fast photoinhibition
Received 8 June 2021

Accepted 11 November 2021

Published online 2 December 2021

${ }^{+}$Corresponding author e-mail: esatyy@utu.fi

Abbreviations: ETR - electron transfer rate; ETR(II) - electron transport rate calculated by taking into account the relative absorption cross-section of PSII; ETR $\mathrm{MAX}_{-}$- maximum electron transport rate; $\mathrm{F}_{\mathrm{v}} / \mathrm{F}_{\mathrm{m}}$ - ratio of variable to maximum fluorescence; $\mathrm{I}_{\mathrm{k}}-$ minimum saturating light intensity; OD - optical density; RLC - rapid light curve; $\alpha$ - light-use efficiency; $\sigma_{\mathrm{II}}(\lambda)$ - wavelength-dependent functional cross-section of PSII.

Acknowledgements: We wish to express our respect for the late Dr. George C. Papageorgiou for his remarkable contribution to photosynthesis research and are honored to be a part of this special issue. This work was supported by the Academy of Finland, grant 33421 (HM and ET), NordAqua (ET, HM), the Vilho, Yrjö, and Kalle Väisälä Foundation (HM), the Emil Aaltonen Foundation (HM), European Union COST Action (TD1102; ET, HM and KBM), and the Ministry of Education, Youth and Sports of CR project 'SustES - Adaptation strategies for sustainable ecosystem services and food security under adverse environmental conditions' (CZ.02.1.01/0.0/0.0/16 019/0000797; to KBM).

Conflict of interest: The authors declare that they have no conflict of interest. 
applications often require a continuously high growth rate. The simplest way to determine the growth rate is to use a batch culture, in which a dilute algal suspension is prepared in a suitable growth medium, usually in a cultivation flask. The culture is stirred, possibly in an atmosphere enriched with $\mathrm{CO}_{2}$, and growth of the algae is followed for a few days until their growth slows down and finally ceases, while cell density becomes very high. If it is possible to discern a phase at which the growth in a very dilute culture is exponential, the maximum specific growth rate is often calculated. In batch growth, the cell density increases during the whole experiment, increasing attenuation of light within the culture. The light attenuation is assumed to be at a minimum during an apparently exponential growth phase, but there is no factual proof for this assumption. In industrial systems, on the other hand, microalgal cultures are often continuously maintained at a constant cell density by frequent additions (influent) of culture medium and simultaneous removal (effluent) of the same amount of culture (see, e.g., Valev et al. 2020). Cell density is commonly followed by measuring the turbidity of the culture, and therefore this cultivation system is called a turbidostat. In a dense culture, light availability is a major factor limiting algal growth. Nevertheless, the turbidostat is usually kept at a relatively high cell density, as a compromise between growth rate and algal concentration, to maximize the productivity of the system (see GarcíaCubero et al. 2021). Due to the higher cell density, mutual shading is typically much stronger in continuous cultures than in a dilute batch. Also, when in excess, light may inhibit growth (see, e.g., Hindersin et al. 2013). In contrast to a batch culture, in a turbidostat, mutual shading does not vary with time, and cells do not need to acclimate to changing light conditions over the growth period of the culture.

In addition to different environmental conditions, growth rates of photosynthetic organisms may vary because of intrinsic differences in their (photosynthetic) characteristics. For example, different organisms have very different capacities to utilize low or high light or to combat stress (Flynn 2009). Photosystem II (PSII) of algae and other photosynthetic organisms is continuously damaged in the light and the damage needs to be constantly repaired, to keep photosynthesis going (for reviews, see Nath et al. 2013, Tyystjärvi 2013). If the rate of the damage is faster than the rate of the repair, e.g., under high light, nonfunctional PSII units accumulate, net photoinhibition occurs, and possibilities to fix carbon may be lost due to the decreased photosynthetic capacity (Raven 2011). In addition, even if an organism manages to compensate the damage by efficient repair, the repair reactions consume a lot of energy (Raven 2011, Miyata et al. 2012, Murphy et al. 2017, Murata and Nishiyama 2018). The vulnerability of PSII to light-induced damage and the repair capacity varies among different algal species (see, e.g., Campbell and Serôdio 2020). Thus, differences in the rates of photoinhibition or in other photosynthetic properties in different algal species may explain, at least partly, their varying growth rates.
In the present study, we set out to investigate whether the maximum specific growth rate measured from a dilute batch culture, photoinhibition tolerance, and/or specific photosynthetic parameters of the algae could predict their growth rates in continuous cultivation. For this, five species of green algae, Scenedesmus obliquus, Scenedesmus ecornis, Desmodesmus quadricauda, Chlorococcum novae-angliae, and Ettlia oleoabundans, were used. These are unicellular freshwater species (except C. novae-angliae, which has been isolated from bog soil) although $S$. obliquus, S. ecornis, and D. quadricauda can also form aggregates, surrounded by a single cell wall (called coenobia), of four or eight cells. These five algal species were cultivated in a phototrophic liquid medium and tested for their growth rates, photoinhibition tolerance, and several other photosynthesis-related processes. To focus on the differences between the growth modes, both the batch and the continuous growth experiments were done as a part of the same experiment, in the same device. We show an inverse relationship between photoinhibition tolerance and the minimum saturating irradiance of PSII. However, based on our results, a continuous culture must be used to measure the growth rate for continuous production.

\section{Materials and methods}

Algal and cyanobacterial strains and culture conditions: S. cf. obliquus (strain \#455 of the collection), S. cf. ecornis (444), and D. quadricauda (463) were obtained from CCALA (Culture Collection of Autotrophic organisms, Třeboň, Czech Republic), and C. novae-angliae (1778) and E. oleoabundans (1185; formerly called also Neochloris oleoabundans) from UTEX (Culture Collection of Algae at the University of Texas at Austin, USA). The algal cultures and the cyanobacterium Synechocystis sp. PCC 6803 were maintained on agar plates made in BG-11 growth medium (Rippka et al. 1979) with $20 \mathrm{mM}$ Hepes- $\mathrm{NaOH}(\mathrm{pH} 7.5)$. Prior to the experiments, if not mentioned otherwise, the algae were grown in $100-\mathrm{ml}$ flasks in BG-11 at the volume of $30 \mathrm{ml}$, at $25-30^{\circ} \mathrm{C}$ with mild shaking in continuous light under the PPFD of $\sim 50 \mu$ mol(photon) $\mathrm{m}^{-2} \mathrm{~s}^{-1}$. Cyanobacteria were grown under similar conditions, but in 250-ml flasks at the volume of $50 \mathrm{ml}$, at $32^{\circ} \mathrm{C}$.

Growth rate measurements: Inocula for the photobioreactor growth experiments were cultured as described above but at $20^{\circ} \mathrm{C}$ at the PPFD of $30 \mu \mathrm{mol}$ (photon) $\mathrm{m}^{-2} \mathrm{~s}^{-1}$ on a shaker set at $100 \mathrm{rpm}$. A $400-\mathrm{ml}$ flat panel photobioreactor (FMT-150, PS Instruments, Czech Republic) was used for growth rate measurements. During the experiments the algal cultures were continuously sparged with a mixture of air and 9,600 ppm $\mathrm{CO}_{2}(1 \%$ $\mathrm{CO}_{2}$ ) at $0.15 \mathrm{ml} \mathrm{min}{ }^{-1}$ flow rate, obtained with a gas mixer unit (GMS 150, PS Instruments). Algae were grown under continuous illumination with white LEDs of the photobioreactor. PPFD was measured from inside of the water-filled bioreactor with a PPFD meter $(L I-250 A$, 
LI-COR, USA) equipped with a submersible sensor (US-SQS/L, Heinz Walz, Germany) and adjusted to the PPFD of $200 \mu \mathrm{mol}$ (photon) $\mathrm{m}^{-2} \mathrm{~s}^{-1}$ (averaged over the panel surface) prior to each experiment. Temperature was maintained at $25^{\circ} \mathrm{C}$. Each experiment consisted of an initial batch phase and a subsequent turbidostat phase. The batch phase was used to calibrate an OD (optical density) 735-nm sensor with measurements of dry algal biomass (see below; Fig. 1S, supplement). OD at $680 \mathrm{~nm}$ was also recorded. The maximum specific growth rate (based on the increase in OD at $735 \mathrm{~nm}$ ) was calculated by finding the best fit (least squares method), during an apparent exponential phase (lasting 15-25 h) of the batch culture, to an exponential equation in Microsoft Excel. In addition, the linear growth rate (when OD at $735 \mathrm{~nm}$ increased linearly with time), observed at a later phase of each batch culture, was calculated at the time when the culture had reached the biomass concentration chosen for the turbidostat mode $\left(\sim 0.43 \mathrm{~g} \mathrm{~L}^{-1}\right)$ by fitting the growth curve (during $7 \mathrm{~h}$ ) to a linear equation in Microsoft Excel.

Turbidostat operation was enabled with the OD 735-nm sensor and a peristaltic pump (PP 500, PS Instruments); fresh BG-11 growth media was pumped into the photobioreactor on demand, in order to dilute the culture and acquire a stable concentration of algal biomass. The dilution rate (which corresponds to the growth rate in the turbidostat) was then measured with a weight balance (CBK 8H, Adam DU, UK) from the harvest (effluent). At the initial part of the turbidostat phase, the OD value at $735 \mathrm{~nm}$ was adjusted, based on dry mass measurements (see below), and then kept stable so that all the cultures were grown at an almost equal biomass concentration of $\sim 0.43 \mathrm{~g} \mathrm{~L}^{-1}$ (Table 1). During a turbidostat run, the growth rate was calculated every six hours, over a time

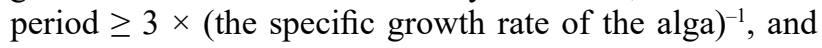
averaged. The photobioreactor was checked daily for biofouling, and when needed, the cultures were mixed mechanically. The biomass concentration was chosen from the 0.1 to $2 \mathrm{~g} \mathrm{~L}^{-1}$ harvest density range often used in biofuel research (for a review, see Su et al. 2017).

Light attenuation by cells inside the photobioreactor was estimated by measuring light intensity, with the LI-COR light meter using the submersible sensor, from separate cultures (pre-grown in flasks as described above) of S. obliquus, C. novae-angliae, and E. oleoabundans. The algal suspensions were placed in a $30-\mathrm{ml}$ cylinder (diameter of $5 \mathrm{~cm}$ ) and light was measured from $1.2 \mathrm{~cm}$ below the illuminated surface (the midway of the $2.4 \mathrm{~cm}$ deep culture in the cylinder, corresponding to the midway of the light path in the photobioreactor). After the first PPFD measurement, a part of the sample was collected for dry mass measurements (see below) to calculate the biomass concentration. After that the algal cultures were diluted with BG-11 to measure light availability inside the culture as a function of cell density. The cyanobacteria were grown only as batch cultures in flasks, as above described, and their growth rate was monitored by measuring OD at $730 \mathrm{~nm}$ with a spectrophotometer from aliquots of the culture. PPFD inside the flasks was measured with the LI-COR light meter using the submersible sensor. Transmission of light through an algal suspension (S. obliquus) was measured with an STS-VIS spectrometer (Ocean Insight).

Dry mass measurements: Samples for dry mass analysis were collected daily from the photobioreactor. Three 5-ml samples were diluted with $0.5 \mathrm{M}$ ammonium formate to a final volume of $10 \mathrm{ml}$ and then filtered through pre-weighed 1.6- $\mu \mathrm{m}$ retention glass fiber filters $(V W R$, USA). Samples were dried for $24 \mathrm{~h}$ at $96^{\circ} \mathrm{C}$ on the filters. After cooling for $1 \mathrm{~h}$ in a desiccator at room temperature, the filters were weighed with an analytical balance (MC1 RC 210-P, Sartorius, Germany). Biomass density was calculated as $\left(\mathrm{m}_{2}-\mathrm{m}_{1}\right)$ /sample volume, where $\mathrm{m}_{1}$ is the mass of the filter and $m_{2}$ is the mass of filter with dried biomass.

Photoinhibition experiments: After 5-20 d of preliminary batch culture in flasks, $35 \mathrm{ml}$ of algal suspension, diluted with fresh BG-11 growth medium to the chlorophyll concentration of $\sim 8 \mu \mathrm{g} \mathrm{ml}{ }^{-1}$, was illuminated at $25^{\circ} \mathrm{C}$ with constant mixing in the PPFDs of $500 \mu \mathrm{mol}($ photon) $\mathrm{m}^{-2} \mathrm{~s}^{-1}$ or $1,500 \mu \mathrm{mol}$ (photon) $\mathrm{m}^{-2} \mathrm{~s}^{-1}$ for $0-90 \mathrm{~min}$ in the absence or in the presence, as indicated, of $0.4 \mathrm{mg} \mathrm{ml}^{-1}$ lincomycin to block the repair of PSII. The cultures used were in the late exponential growth phase or linear growth phase. In some cases, as indicated, the algae were let to recover after the light treatment for two days at their growth conditions (in the light). Chlorophylls (Chl) $a$ and $b$ were extracted from separate cultures by at least overnight incubation in methanol at $4^{\circ} \mathrm{C}$ in the dark and quantified according to Inskeep and Bloom (1985); the extraction was considered complete if the pellet had no green tint after centrifugation. PSII activity was assayed by measuring the $\mathrm{Chl} a$ fluorescence parameter variable to maximum fluorescence $\mathrm{F}_{\mathrm{v}} / \mathrm{F}_{\mathrm{m}}=\left(\mathrm{F}_{\mathrm{m}}-\mathrm{F}_{0}\right) / \mathrm{F}_{\mathrm{m}}$ with Multi-Color-PAM fluorometer (measuring beam: $625 \mathrm{~nm}$; Heinz Walz, Effeltrich, Germany) after 10 min in darkness. Minimum fluorescence, $\mathrm{F}_{0}$, was measured using only very low intensity measuring flashes, while $\mathrm{F}_{\mathrm{m}}$ was induced with 800-ms saturating pulses (PPFD of 10,000 $\mu \mathrm{mol} \mathrm{m} \mathrm{m}^{-2} \mathrm{~s}^{-1}$.

Rapid light curves (RLCs) were measured from all species using the Multi-Color PAM fluorometer (Heinz Walz). All cultures used in RLC measurements were in the late exponential growth phase, and samples from batch cultures (in flasks) were normalized to OD at $680 \mathrm{~nm}=0.2$, to obtain similar, healthy algal samples, by diluting with fresh BG-11 growth medium. OD at $680 \mathrm{~nm}$ corresponds to the peak of Chl $a$ absorption and gives a rough estimate of the Chl concentration. After dilution, the samples were acclimatized for $15 \mathrm{~min}$ in the growth conditions (in the light) prior to the measurements. The measuring protocol consisted of 13 steps of increasing intensities of white light, up to PPFD of $1,115 \mu \mathrm{mol} \mathrm{m} \mathrm{m}^{-2} \mathrm{~s}^{-1}$, with each step lasting for $90 \mathrm{~s}$. The intensity of the 440-nm measuring 
light (modulation frequency $20,000 \mathrm{~Hz}$ ) was adjusted for each species separately to achieve comparable fluorescence values. The temperature was maintained at $27^{\circ} \mathrm{C}$ using the temperature control unit (US-T) accessory of Multi-Color $P A M$. The samples were agitated with a magnetic stirrer during the measurements. The parameters $\alpha$ (light-use

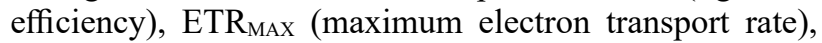
and $I_{k}$ (minimum saturating irradiance) were derived by analyzing the RLCs according to the model described by Eilers and Peeters (1988) using the in-built fitting protocol of the PamWin-3 (v3.22) software of Multi-Color PAM.

Wavelength-dependent functional cross-sections of PSII $\left[\sigma_{\text {II }}(\lambda)\right]$ were measured as described by Schreiber et al. (2012) from samples that had been dark-acclimated for $15 \mathrm{~min}$ but were otherwise identical to the ones used in the RLC measurements. This method allowed the estimation of photons absorbed by PSII vs. photons absorbed by all photosynthetic pigments [PPFD(II)] and, therefore, also the estimation of PSII-specific (absolute) electron transport rate $[\mathrm{ETR}(\mathrm{II})]$. The $\sigma_{\mathrm{II}}(\lambda)$ values obtained with a combination of white light and 440-nm measuring light were used to calculate PPFD(II) and ETR(II) during the RLC measurements in all algae strains using the PamWin-3 software.

$\mathbf{Q}_{\mathbf{A}^{-}}$reoxidation: Kinetics of $\mathrm{Q}_{\mathrm{A}^{-}}$reoxidation were measured with an FL 200 fluorometer with a SuperHead optical 525 unit (PS Instruments) after a strong single turnover flash $(530 \mathrm{~nm})$. Prior to the measurements, the algae were diluted to OD at $680 \mathrm{~nm}=0.3$ with fresh BG-11 growth medium and dark-acclimated for $5 \mathrm{~min}$. The first measuring point was recorded $150 \mu$ s after the flash. The decay of Chl $a$ fluorescence yield (f) was fitted to the equation $\mathrm{f}(\mathrm{t})=\mathrm{a} \times \exp (-\mathrm{b} \times \mathrm{t})+\mathrm{c} \times \exp (-\mathrm{d} \times \mathrm{t})+\mathrm{g} \times$ $\exp (-\mathrm{h} \times \mathrm{t})$, where $\mathrm{t}$ is time, in SigmaPlot (Systat Software, Inc.) to calculate the rate constants for electron transfer from $\mathrm{Q}_{\mathrm{A}}$ to $\mathrm{Q}_{\mathrm{B}}(\mathrm{b})$, an apparent first-order rate constant for $\mathrm{PQ}$ binding to an empty $\mathrm{Q}_{\mathrm{B}}$ site and possible $\mathrm{PQH}_{2}$ release (d) and recombination of $\mathrm{S}_{2} \mathrm{Q}_{\mathrm{A}^{-}}$to $\mathrm{S}_{1} \mathrm{Q}_{\mathrm{A}}(\mathrm{h})$, and the proportions of functional PSII units (a), PSII units with an empty $\mathrm{Q}_{\mathrm{B}}$ site $(\mathrm{c})$, and $\mathrm{Q}_{\mathrm{B}}$ nonreducing PSII reaction centers $(\mathrm{g})$.

\section{Results}

\section{Fast batch growth rate did not always predict fast continuous growth}

Growth rates of five green algal species at moderate light [200 $\mu \mathrm{mol}$ (photon) $\mathrm{m}^{-2} \mathrm{~s}^{-1}$ ] were compared by cultivating them in a flat panel photobioreactor, first as a batch culture, and then in a continuous mode (turbidostat) where the biomass concentration was kept constant (at $\sim 0.43 \mathrm{~g} \mathrm{~L}^{-1}$ ) by frequent replacement of part of the culture with fresh growth medium. Growth was followed by recording optical density (OD) at $735 \mathrm{~nm}$. Relatively little variation was observed in the growth rates of the different algal species during the turbidostat mode (Fig. 1, Table 1); E. oleoabundans, C. novae-angliae, and S. ecornis had almost the same duplication rates $\left(0.68,0.71\right.$, and $0.72 \mathrm{~d}^{-1}$, respectively), whereas the growth rates of the other two species, D. quadricauda and $S$. obliquus, were slightly higher ( 0.85 and $0.94 \mathrm{~d}^{-1}$, respectively). In the batch mode, on the other hand, the growth rates of the algae differed more from each other; the maximum specific growth rate, measured during an initial exponential growth phase, of D. quadricauda was approximately three times as high as that of E. oleoabundans (Fig. 1, Table 1).

It was not feasible to measure how light availability is changing inside the used flat panel photobioreactor during algal growth; therefore, light intensity inside an optically similar setting was measured from different dilutions of E. oleoabundans, C. novae-angliae, and S. obliquus (Fig. 2A). Unexpectedly, light that passed through an $S$. obliquus culture was enriched in green $(\sim 520-580 \mathrm{~nm})$ and far-red wavelengths (Fig. $2 B$ ), as these wavelengths are not well absorbed by chlorophylls. We also measured light intensity inside $250-\mathrm{ml}$ flasks during a $50-\mathrm{ml}$ cyanobacterial batch culture. In both cases, light availability decreased drastically when cell density increased (Fig. 2). The effects of cell density on light availability slightly differed in the different organisms, probably due to different cell sizes, as the used flat panel bioreactor had a relatively short light path of $24 \mathrm{~mm}$, which is rather close to what the 50-ml cyanobacterial cultures experienced in the $250-\mathrm{ml}$ flasks. Based on the light availability measurements (Fig. $2 A$ ), it is clear that during the turbidostat culture the algae received less light than a dilute culture (at its exponential growth phase). Consequently, it is unsurprising that the specific growth rates measured during the exponential batch growth were higher than the growth rates during the turbidostat mode (Fig. 1F). However, these growth rate measurements also did not correlate very well (Fig. $1 F ; \mathrm{r}^{2}=0.608$ ) with each other, as different species grew fast during the batch and different ones during the continuous growth.

However, to achieve a fairer comparison for batch and turbidostat growth modes, growth rates in the batch culture were calculated (by linear fitting) also during the phase when the algae had achieved the biomass concentration selected for turbidostat mode $\left(\sim 0.43 \mathrm{~g} \mathrm{~L}^{-1}\right)$; at that point the increase in the algal concentration in the batch culture was rather linear. The change in the concentration of $S$. obliquus at that point, however, slightly resembled an exponential increase, which may partially explain its high turbidostat growth rate. However, even in this comparison the batch and turbidostat growth rates did not correlate (Fig. $1 F$, Table $1 ; \mathrm{r}^{2}=0.038$ ), not even for S. obliquus.

\section{Susceptibility to photoinhibition varied among algal species but showed no general correlation with the growth rate}

To see if susceptibility to photoinhibition of PSII could explain the differences in the growth rates, cells of the five green algal species were illuminated with high light [PPFD of $500 \mu$ mol(photon) $\mathrm{m}^{-2} \mathrm{~s}^{-1}$ ] in the presence of lincomycin, an antibiotic that blocks translation in 


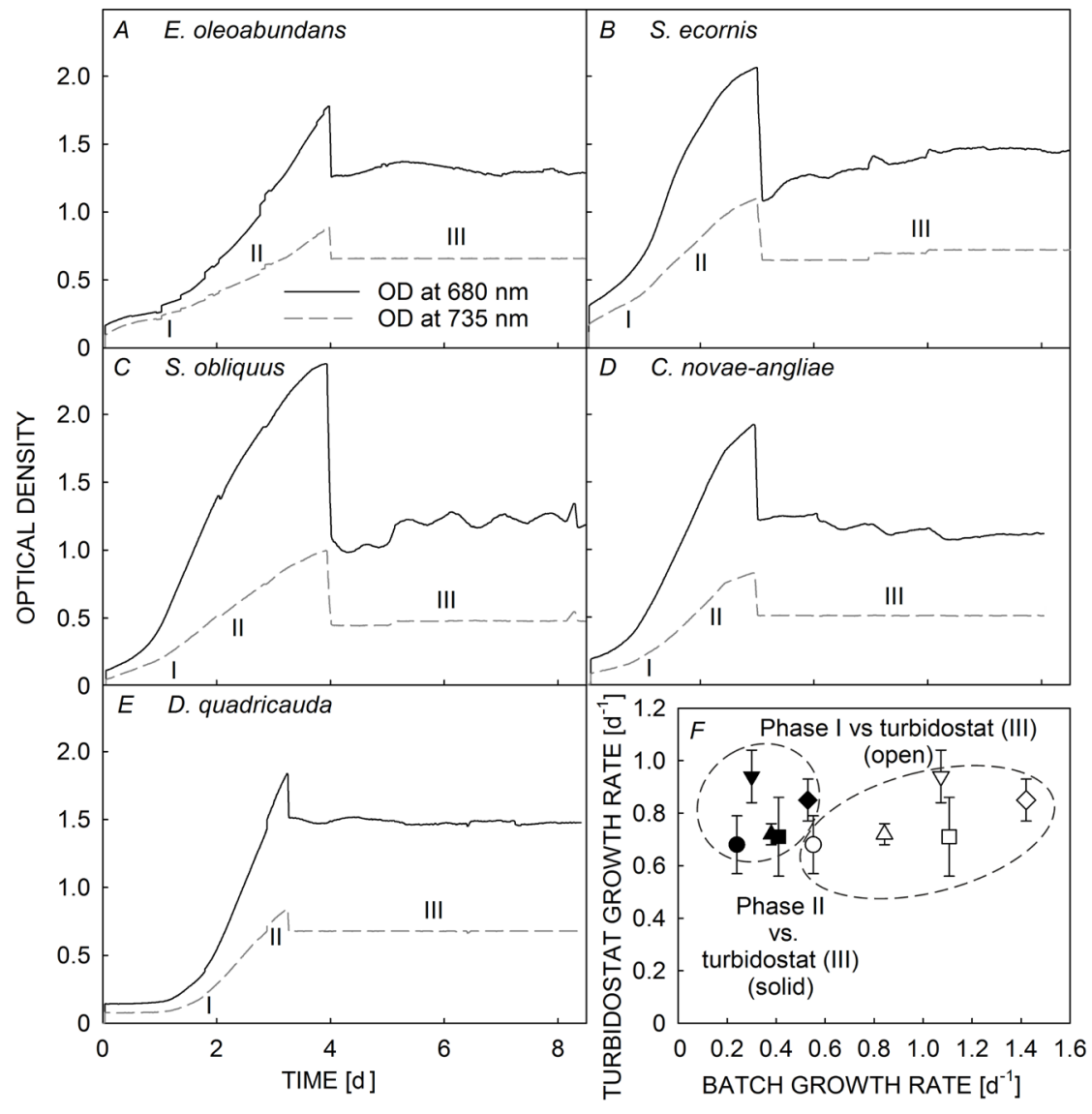

Fig. 1. Growth of five species of green algae in a flat panel photobioreactor. $(A-E)$ The indicated algae were grown first in batch mode and then in turbidostat mode [the change between the growth modes is seen as a drop in both the optical density (OD) values at the $3^{\text {rd }}$ or $4^{\text {th }}$ growth day], where biomass concentration was kept constant by dilutions with fresh growth medium. Different growth phases are highlighted with Roman numerals; I: an initial exponential growth phase in batch mode, II: a linear growth phase in batch mode, III: turbidostat mode. Growth was measured by recording OD of the culture at 680 and $735 \mathrm{~nm}$. $(F)$ Comparison of growth rates during the batch and turbidostat modes. The batch growth rate was measured during an initial exponential growth phase (phase I, open symbols) and during a linear growth phase, i.e., when the algae had reached the same biomass concentration as used in the turbidostat mode (phase II, solid symbols), from Ettlia oleoabundans (circles), Scenedesmus ecornis (upward triangles), Scenedesmus obliquus (downward triangles), Chlorococcum novae-angliae (squares), and Desmodesmus quadricauda (diamonds). Turbidostat growth rate measurements $(F)$ are averaged over a single photobioreactor experiment (growth rate calculations were conducted every $6 \mathrm{~h}$ ). Samples within the same encirclement can be compared with each other. Error bars show SD.

Table 1. Biomass concentrations and growth rates during continuous (turbidostat) growth, and batch growth, at the PPFD of $200 \mu \mathrm{mol}$ $\mathrm{m}^{-2} \mathrm{~s}^{-1}$ at $25^{\circ} \mathrm{C}$ of five species of green algae. SD values are calculated from turbidostat growth rate measurements, conducted every $6 \mathrm{~h}$ over a single photobioreactor experiment. Growth rates during batch were measured during an initial exponential growth in a dilute culture (maximum specific growth rate) and during the time point the algae had reached the concentration used for the turbidostat mode (when the algal concentration increased linearly).

\begin{tabular}{lllll}
\hline Species & $\begin{array}{l}\text { Turbidostat } \\
\text { Biomass }\left[\mathrm{g} \mathrm{L}^{-1}\right]\end{array}$ & Growth rate $\left[\mathrm{d}^{-1}\right]$ & $\begin{array}{l}\text { Batch } \\
\text { Growth rate, } \\
\text { exponential phase }\left[\mathrm{d}^{-1}\right]\end{array}$ & $\begin{array}{l}\text { Growth rate, } \\
\text { linear phase }\left[\mathrm{d}^{-1}\right]\end{array}$ \\
\hline Ettlia oleoabundans & $0.434 \pm 0.010$ & $0.68 \pm 0.11$ & 0.55 & 0.24 \\
Scenedesmus ecornis & $0.426 \pm 0.004$ & $0.72 \pm 0.04$ & 0.84 & 0.38 \\
Scenedesmus obliquus & $0.434 \pm 0.012$ & $0.94 \pm 0.10$ & 1.07 & 0.30 \\
Chlorococcum novae-angliae & $0.420 \pm 0.005$ & $0.71 \pm 0.15$ & 1.11 & 0.41 \\
Desmodesmus quadricauda & $0.421 \pm 0.007$ & $0.85 \pm 0.08$ & 1.42 & 0.53 \\
\hline
\end{tabular}



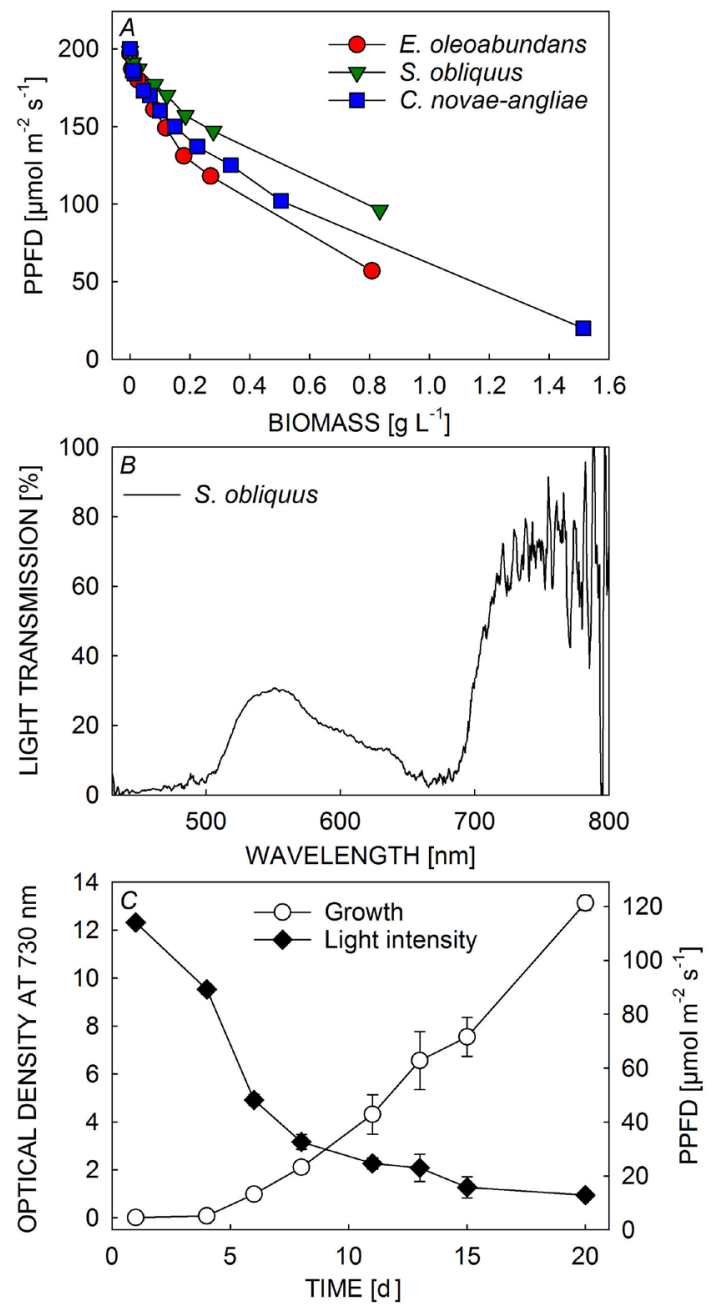

Fig. 2. Effects of cell density on light availability inside cultures of photosynthetic organisms. (A) PPFD values at $1.2 \mathrm{~cm}$ depth inside a cylinder filled with different dilutions of cultures of the indicated green algae. Biomass concentrations were calculated from dry mass measurements of the undiluted samples (three technical repetitions, error bars are smaller than the symbols). (B) A spectrum of light transmitted through an algal (Scenedesmus obliquus) culture (2.4-cm light path) with a biomass concentration of $0.834 \pm 0.008 \mathrm{~g} \mathrm{~L}^{-1}$ [the highest cell density shown in $(A)$ ]. (C) Light intensity (black symbols) inside $50-\mathrm{ml}$ cyanobacterial batch cultures after 1 to $20 \mathrm{~d}$ of growth (open symbols). Error bars show SD from three experimental repetitions.

chloroplasts and thereby inhibits the repair of damaged PSII units. A relatively high light intensity, instead of the growth light intensity, was used to observe clear photoinhibition. The direct proportionality between light intensity and the rate constant of photoinhibition (for a review, see Tyystjärvi 2013) guarantees that the results predict photoinhibition well at lower light intensities, too. Photoinhibition of PSII was monitored with the $F_{v} / F_{m}$ parameter; a decrease in $\mathrm{F}_{\mathrm{v}} / \mathrm{F}_{\mathrm{m}}$ is known to correlate well with the loss of oxygen evolution in photoinhibition experiments (for a review, see Tyystiärvi 2013). Clear differences in the rates of PSII photoinhibition were observed: after the 40-min illumination, 89, 80, 76, 54, and $39 \%$ of PSII remained active in S. ecornis, D. quadricauda, $S$. obliquus, C. novae-angliae, and E. oleoabundans, respectively (Fig. 3A). E. oleoabundans, the slowest grower in both the batch and turbidostat cultivation modes (Table 1), was indeed highly susceptible to photoinhibition of PSII, and D. quadricauda, the alga that was the most tolerant against the high-light-induced inactivation of PSII, exhibited high batch growth rates (but not the highest growth rate in the turbidostat mode; Table 1). However, only a weak correlation between photoinhibition and growth rates, in either batch or continuous culture, was observed $\left(\mathrm{r}^{2}=0.25-0.28\right.$; Fig $\left.3 B\right)$. The susceptibility of the algae to the damaging reaction of photoinhibition of PSII did not seem to be a decisive factor in determining the growth rate here.

If lincomycin did not completely inhibit the repair of damaged PSII units in the used algal species, the observed photoinhibition rates would not have been comparable. Therefore, we tested whether lincomycin blocked PSII repair in the used algal species (Fig. 3C). In all the cases, the $F_{v} / F_{m}$ values increased when the algae were let to recover for two days at their growth light [PPFD of $40 \mu \mathrm{mol}$ (photon) $\mathrm{m}^{-2} \mathrm{~s}^{-1}$ ] after a strong high light illumination [PPFD of $1,500 \mu \mathrm{mol}$ (photon) $\mathrm{m}^{-2} \mathrm{~s}^{-1}$ for $90 \mathrm{~min}$ ] in the presence of lincomycin. However, the recovery was much faster and essentially complete in the absence of lincomycin (tested in all the algal species except $D$. quadricauda), indicating that the algae were not resistant to lincomycin (Fig. 3D). It has been shown that $F_{m}$ is not reached simply by the reduction of the $Q_{A}$ electron acceptor of PSII, but formation of a distinctive light-adapted closed state of PSII reaction center, formed via conformational changes in PSII, is required to obtain $\mathrm{F}_{\mathrm{m}}$ (Sipka et al. 2021). The finding that the $\mathrm{F}_{\mathrm{v}} / \mathrm{F}_{\mathrm{m}}$ values of PSII preparations isolated from healthy plant leaves or cyanobacteria are largely determined by a conformational change of the PSII reaction center may suggest that the lowering of $F_{v} / F_{m}$ in photoinhibition is partly caused by a conformational change and partly by lowering of the photochemical efficiency of PSII. Therefore, an increase in $\mathrm{F}_{\mathrm{v}} / \mathrm{F}_{\mathrm{m}}$ after photoinhibition in the presence of lincomycin (Fig. 3C) may not indicate an increase in PSII yield.

\section{Light utilization capabilities and PSII electron transfer kinetics differed between the algae}

To understand the limitations behind algal growth, we analyzed photosynthetic reactions of the algae by $\mathrm{Chl} a$ fluorescence measurements. The five green algae were illuminated with stepwise increasing intensities of white light, to record light-response curves (Fig. 4A). Based on the measurements, E. oleoabundans, S. ecornis, and C. novae-angliae were observed to have slightly higher light-use efficiencies $(\alpha$; measured as the slope of the initial linear rise in the relative electron transfer rate in low light) than D. quadricauda or S. obliquus. E. oleoabundans had both the highest maximum (relative) electron transport rate $\left(\mathrm{ETR}_{\mathrm{MAX}}\right)$ and minimum saturating irradiance $\left(\mathrm{I}_{\mathrm{k}}\right)$ and D. quadricauda the lowest ones (Fig. 4B). In all the 

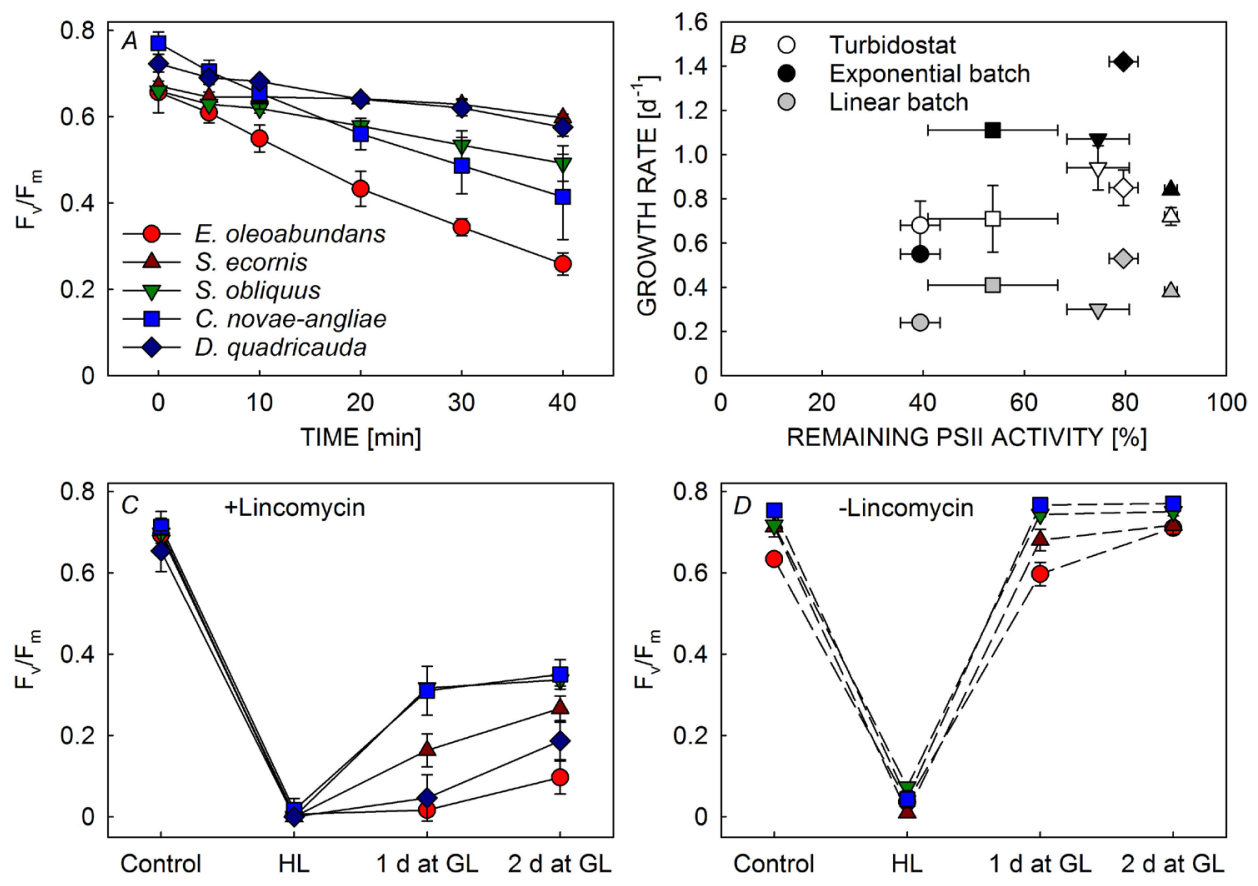

Fig. 3. Photoinhibition of PSII. ( $A$ ) Changes in $\mathrm{F}_{\mathrm{v}} / \mathrm{F}_{\mathrm{m}}$, measured from five species of green algae during 0-40 min high light illumination [PPFD of $500 \mu \mathrm{mol}\left(\right.$ photon) $\mathrm{m}^{-2} \mathrm{~s}^{-1}$ ] at $25^{\circ} \mathrm{C}$ in the presence of lincomycin. $\mathrm{F}_{\mathrm{v}} / \mathrm{F}_{\mathrm{m}}$ values were measured after 10 min of darkness. Error bars, shown when larger than the symbol, show SD calculated from at least three experimental repetitions. $(B)$ Growth rates of the algae measured during continuous culture (turbidostat; open symbols), and during batch culture, either during an initial exponential growth (solid symbols) or during a linear growth phase (when the algae had reached the same biomass concentration as used in the turbidostat mode; grey symbols), plotted against photoinhibition tolerance (remaining PSII activity after the 40-min illumination). The symbol shapes are the same as in $(A)$. Growth data are from Fig. 1. (C) The algae were illuminated for 90 min, at the PPFD of $1,500 \mu \mathrm{mol}($ photon $) \mathrm{m}^{-2} \mathrm{~s}^{-1}$ at $25^{\circ} \mathrm{C}$ (HL, high light), in the presence of lincomycin. After the high light, the algae were let to recover for $2 \mathrm{~d}$ at their growth conditions [GL, growth light; PPFD $40 \mu \mathrm{mol}$ (photon) $\mathrm{m}^{-2} \mathrm{~s}^{-1}$. (D) The algae (except Desmodesmus quardicauda) were illuminated as in $(C)$ but in the absence of lincomycin. $\mathrm{F}_{\mathrm{v}} / \mathrm{F}_{\mathrm{m}}$ values were measured after 20 min of darkness. The symbol shapes and colours are the same as in $(A)$. Error bars, shown when larger than the symbol, show SD calculated from three experimental repetitions.

wavelengths measured, E. oleoabundans, S. ecornis, and D. quadricauda had bigger functional cross-sections of PSII $\left[\sigma_{\mathrm{II}}(\lambda)\right]$ than $S$. obliquus and $C$. novae-angliae (Fig. $4 C$ ). In line with the low $\sigma_{\text {II }}(\lambda)$, PSII specific electron transfer rates [taking into account the measured values for absorption cross-sections; ETR(II)] of C. novae-angliae were low (Fig. 4D) compared to the relative electron transport rates of the same species (Fig. 4A).

The measured light-use efficiencies ( $\alpha$ parameter) did not, in general, correlate either with electron transfer rates, or with growth rates. Neither did the functional cross-sections of PSII correlate with the other measured photosynthetic parameters. Instead, minimum saturating irradiances (and to a lesser degree, ETR $\mathrm{ETAX}_{\mathrm{MA}}$ correlated negatively with photoinhibition tolerance of the species $\left(\mathrm{r}^{2}=0.86\right.$; Fig. $\left.4 E\right)$. However, a low minimum saturating irradiance of an alga did not lead to a high continuous growth rate $\left(\mathrm{r}^{2}=0.13\right.$; Fig. $\left.4 F\right)$. Relatively low correlations were found also between minimum saturating irradiance and batch growth rate during the exponential $\left(r^{2}=0.40\right)$ or the linear $\left(r^{2}=0.59\right)$ growth phases.
To understand if the correlation of low minimum saturating irradiance for electron transfer rate with photoinhibition tolerance or growth rate could be explained by electron transfer kinetics of PSII, reoxidation of $\mathrm{Q}_{\mathrm{A}}^{-}$ (an electron acceptor of PSII) was measured from the algae after a short dark-acclimation to oxidize $\mathrm{Q}_{\mathrm{A}}$ (Fig. 5). The $\mathrm{Q}_{\mathrm{A}}^{-}$reoxidation kinetics in $S$. obliquus and $C$. novaeangliae were visually different from the kinetics in the other three algae (D. quadricauda, E. oleoabundans, and $S$. ecornis). The data were fitted to a model with three exponential components corresponding to $\mathrm{Q}_{\mathrm{A}}{ }^{-} / \mathrm{Q}_{\mathrm{B}} \rightarrow$ $\mathrm{Q}_{\mathrm{A}} / \mathrm{Q}_{\mathrm{B}}{ }^{-}$electron transfer (fast component), binding of a plastoquinone (PQ) molecule to an empty $\mathrm{Q}_{\mathrm{B}}$ site, possibly including also the release of $\mathrm{PQH}_{2}$ from the $\mathrm{Q}_{\mathrm{B}}$ site (middle component), and charge recombination of $\mathrm{S}_{2} \mathrm{Q}_{\mathrm{A}}{ }^{-} \rightarrow \mathrm{S}_{1} \mathrm{Q}_{\mathrm{A}}$, occurring in inactive PSII centers (slow component). The results show that the binding of PQ to PSII or the release of $\mathrm{PQH}_{2}$ was slow in $S$. obliquus and $C$. novae-angliae, compared to the other algae, and the amount of inactive, $\mathrm{Q}_{\mathrm{B}}$ nonreducing PSII centers was high in these two algae (Table 2). 

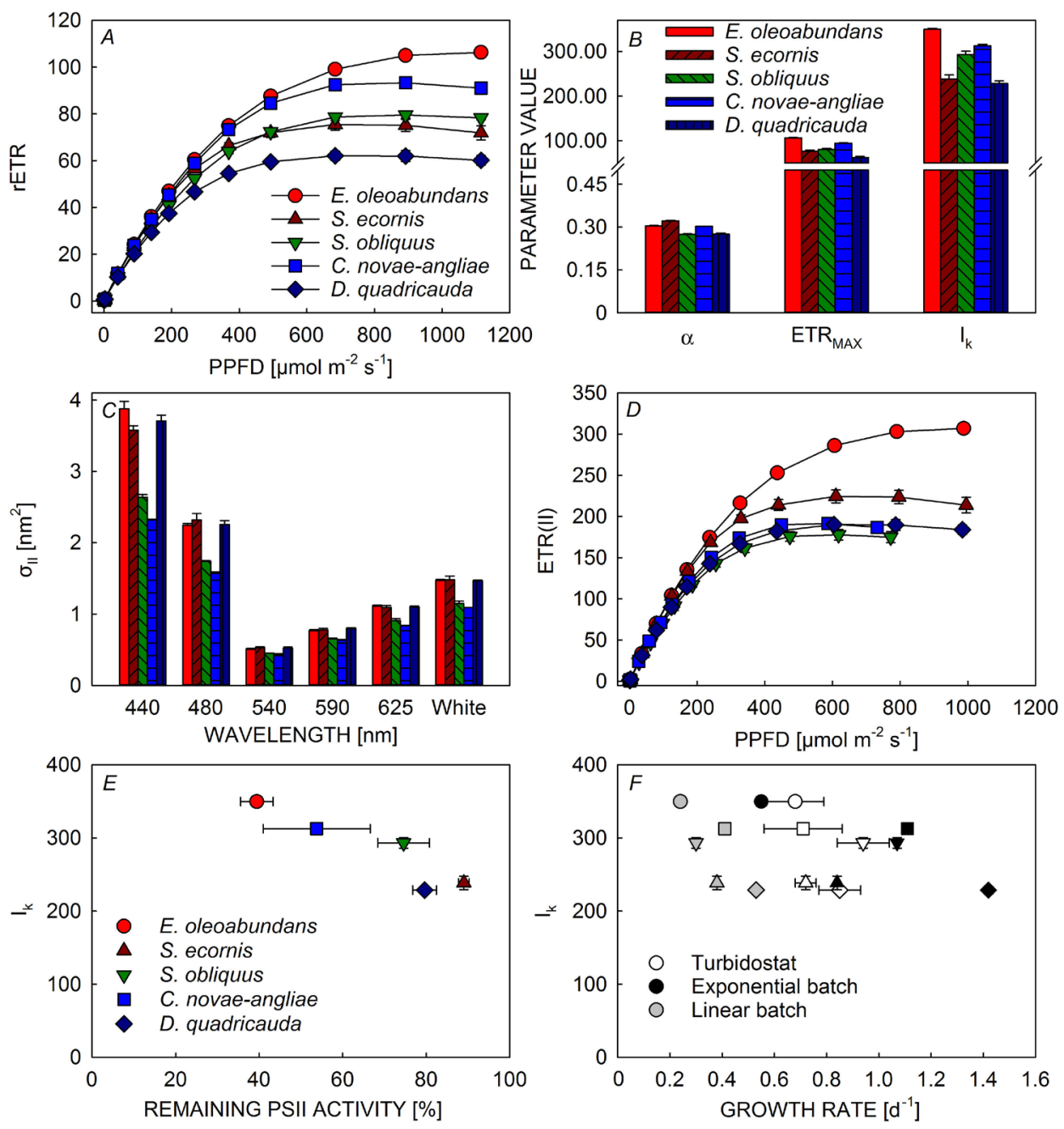

Fig. 4. Photosynthetic parameters in five species of green algae. (A) Relative electron transport rates (rETR) were measured by illuminating light-acclimated algal suspension for $90 \mathrm{~s}$ with the indicated intensity of light before firing a saturating pulse. $(B)$ Parameters

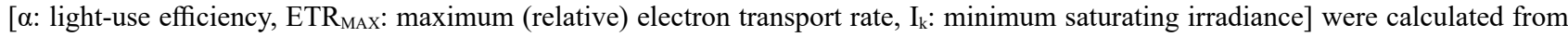
the light curves. $(C)$ Wavelength-dependent functional cross-sections of PSII $\left[\sigma_{\mathrm{II}}(\lambda)\right]$ were measured from dark-acclimated algae. $(D)$ PSII specific electron transport rates [ETR(II)] were calculated by using rETR data from $(A)$ and the white light specific $\sigma_{\text {II }}$ values from $(C)$. All data points are averages from three experimental repetitions and error bars, shown when larger than the symbol, indicate SD. $(E, F)$ Comparison of $\mathrm{I}_{\mathrm{k}}$ with photoinhibition tolerance $(E)$ and growth rates $(F)$ of the five algae. Growth rates of the algae were measured during continuous culture (turbidostat; open symbols), and during batch culture, either during initial exponential growth (solid symbols) or during linear growth (when the algae had reached the same biomass concentration as used in the turbidostat mode; grey symbols), the symbol shapes and colours $(E)$ are the same as in $(A-E)$. Growth data are from Fig. 1.

\section{Discussion}

\section{Why do growth rates differ between batch and turbidostat modes?}

The maximum specific growth rate, representing the highest cell division rate the organism is capable of, is usually (as well as here) calculated based on a dilute batch culture. However, to select the most suitable species for a biotechnological application that uses continuous culture, a test with batch culture may not reveal the fastest growing candidates; we observed poor correlations between growth rates in batch and turbidostat cultures in the tested five green algal species S. obliquus, S. ecornis, D. quadricauda, C. novae-angliae, and E. oleoabundans (Fig. 1). It has been proposed that even measurements of the maximum specific growth rate would be more reliable if done in a continuous culture (Barbera et al. 2019). However, the conditions selected for our turbidostat cultivation would not reveal the maximum rate of cell division.

The observation that one species excels (regarding its growth rate) in a batch culture and a different species excels in a continuous culture, may not be surprising. An obvious difference between the growth modes is that during the initial phase of batch culture, the algae experience (continuous) high irradiances when there is 
little shading by other cells (see Fig. 2). E. oleoabundans grew slowly in the batch mode but showed an average growth rate in the turbidostat mode (Fig. 1). The high sensitivity of this species to photoinhibition of PSII (Fig. 3) may have been less important during continuous culture at a high cell density (and consequently lower light intensity) than at the beginning of the batch. Accordingly, algae cultured in an outdoor bioreactor were more prone to photoinhibition when their concentration was kept low (Hindersin et al. 2013). In general, attenuation of light by the antenna system of the organism itself or by a thick suspension of microalgae strongly protect against photoinhibition (Pätsikkä et al. 2002, Serôdio and Campbell 2021). Therefore, although we did not observe a general correlation between photoinhibition tolerance and growth rate (Fig. $3 B$ ), susceptibility to photoinhibition may be expected to limit growth under certain conditions, at least in certain species.

Small antenna size is a common acclimation response to high light (e.g., Neale and Melis 1986, Treves et al. 2016). Therefore, the observed small PSII cross-sections of $S$. obliquus and C. novae-angliae (Fig. 4C) may have contributed to their relatively fast batch growth rates (Table 1). However, the slow forward electron transfer in these two algae (Fig. 5) may have affected the calculations

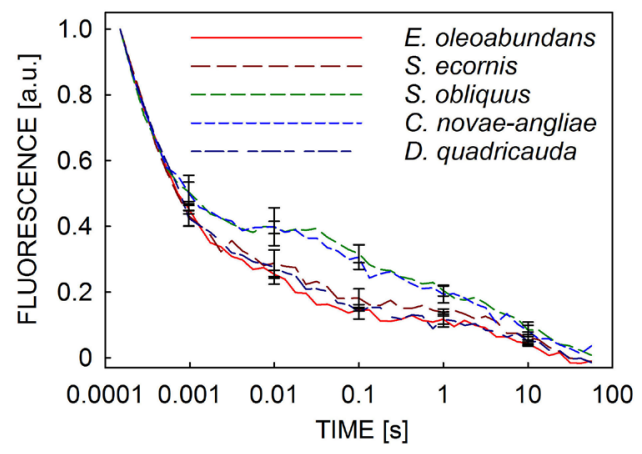

Fig. 5. $\mathrm{Q}_{\mathrm{A}}^{-}$reoxidation kinetics in five species of green algae. Dark-acclimated algae were given a single-turnover saturating flash, after which the decay in fluorescence yield was recorded (first data point $150 \mu \mathrm{s}$ after the flash). To facilitate comparison, the lines are normalized to the first data point. The lines show averages from three experimental repetitions and error bars show SD. of the cross-sections (which employs $\mathrm{Q}_{\mathrm{A}}^{-}$reoxidation parameters; Schreiber et al. 2012).

In a dense continuous culture, the ability to utilize all light efficiently may be more important than during batch growth. However, as D. quadricauda and S. obliquus, the two fastest growers under the turbidostat mode (Table 1), had the lowest light-use efficiencies (Fig. 4B), it seems reasonable to assume that under our conditions, the biomass concentration during the turbidostat mode was low enough to prevent serious limitations in light availability. The fact that, in general, we only found very weak correlations between electron transfer parameters and growth rates (Fig. 4F) may not be surprising. Even though the fluorescence parameters usually correlate well with carbon fixation in different algae (Barranguet and Kromkamp 2000), the assimilated carbon may be used, instead of growth, to synthesize different (secondary) metabolites or storage compounds.

Dilute and dense cultures (whether batch or turbidostat) are also not directly comparable due to the fact that in a dilute culture, each algal cell continuously receives the same light intensity (at least when the light path is relatively short) while in a dense culture, mixing exposes the cells to rapidly changing light conditions, which has been shown to affect algal growth (Grobbelaar et al. 1996, Levasseur et al. 2018). In our case, however, growth rates even between dense batch and turbidostat cultures showed a weak correlation (Fig. $1 F$ ), although both culture types were mixed similarly.

Besides the difference in the attenuation of light, the growth medium develops differently during a batch than during a continuous growth experiment and many of these differences are likely to be strongly dependent on the experimental conditions and species studied. In the batch mode, main nutrients are usually consumed in a few days (e.g., Valev et al. 2020), whereas in the turbidostat mode, the growth medium is continuously replenished. Depletion of nutrients may partially explain why the batch growth rates during the time the algae had reached the concentration that was later used for the turbidostat mode were slower than the growth rates during the turbidostat cultivation phase (Fig. $1 F$ ). The different nutrient contents in batch and turbidostat modes may also favor different algal species. However, Valev et al. (2020) observed that even though nutrient contents in the effluent were stable during a turbidostat run, ammonium contents were (constantly) low, compared to the influent, and no

Table 2. Kinetic parameters of $\mathrm{Q}_{\mathrm{A}}^{-}$reoxidation in five species of green algae, calculated by fitting the data shown in Fig. 5 to a model with three exponential components.

\begin{tabular}{lllllll}
\hline Species & $\begin{array}{l}\mathrm{Q}_{\mathrm{A}}{ }^{-} \rightarrow \mathrm{Q}_{\mathrm{B}}^{-} \\
{[\mu \mathrm{s}]}\end{array}$ & $\begin{array}{l}\mathrm{PQ} \text { binding/PQH } \\
\text { release [ms] }\end{array}$ & $\begin{array}{l}\mathrm{S}_{2} \mathrm{Q}_{\mathrm{A}}{ }^{-} \rightarrow \\
\mathrm{S}_{1} \mathrm{Q}_{\mathrm{A}}[\mathrm{s}]\end{array}$ & $\begin{array}{l}\text { Functional PSII } \\
{[\%]}\end{array}$ & $\begin{array}{l}\text { PSII with an empty } \\
\mathrm{Q}_{\mathrm{B}} \text { site [\%] }\end{array}$ & $\begin{array}{l}\mathrm{Q}_{\mathrm{B}} \text { nonreducing } \\
{[\%]}\end{array}$ \\
\hline Ettlia oleoabundans & 380 & 15 & 7.4 & 71 & 18.5 & 10.5 \\
Scenedesmus ecornis & 352 & 24 & 9.6 & 72 & 15 & 13 \\
Scenedesmus obliquus & 312 & 138 & 12.4 & 67 & 15 & 17 \\
Chlorococcum novae-angliae & 330 & 84 & 9.8 & 67 & 15.5 & 17.5 \\
Desmodesmus quadricauda & 410 & 27 & 9.6 & 71 & 18 & 11 \\
\hline
\end{tabular}


phosphate was detected in the effluent, indicating efficient nutrient uptake by algae cultured in a turbidostat.

\section{What is the relationship between PSII photoinhibition and growth rate during continuous culture?}

Some of the green algae tested here seemed very vulnerable to PSII damage while others were considerably more resistant (Fig. 3A). However, these differences did not in general lead to different growth rates in continuous culture (Fig. 3B). The highest light intensity at which a phototroph (an alga in this case) gains energy (i.e., the highest intensity at which the costs of PSII repair do not exceed the energy that can be obtained by photosynthesis) depends on the species (Murphy et al. 2017). In addition, acclimation allows for example the green alga Chlamydomonas reinhardtii to grow at the extreme PPFD of 3,000 $\mu \mathrm{mol}$ (photon) $\mathrm{m}^{-2} \mathrm{~s}^{-1}$ (Virtanen et al. 2021). It is concluded that all the tested algal species were able to grow under the light intensity used in the present study [200 $\mu \mathrm{mol}$ (photon) $\mathrm{m}^{-2} \mathrm{~s}^{-1}$. A Scenedesmus species has previously been shown to possess an efficient repair cycle and to be able to maintain almost all PSII units functional even at $500 \mu \mathrm{mol}($ photon) $\mathrm{m}^{-2} \mathrm{~s}^{-1}$ (Komenda 1998), indicating that most probably the algae here did not experience net photoinhibition during the continuous culture. Also, here most of the algal species were able to repair their PSII units in less than $24 \mathrm{~h}$, after a high light illumination that had led to a complete loss of PSII activity (Fig. 3D).

The efficient repair cycle indicates that at the PPFD of $500 \mu \mathrm{mol}$ (photon) $\mathrm{m}^{-2} \mathrm{~s}^{-1}$ it is beneficial to invest the energy to keep PSII functional (rather than to wait for better times). Indeed, $500 \mu \mathrm{mol}$ (photon) $\mathrm{m}^{-2} \mathrm{~s}^{-1}$ is oversaturating for all the studied algae (Fig. $4 B$ ); when (light) energy is not a limiting factor, it can be used to constantly repair PSII. The same situation may apply to the continuous culture here, where the used PPFD of $200 \mu \mathrm{mol}($ photon) $\mathrm{m}^{-2} \mathrm{~s}^{-1}$, was close to saturating (Fig. $4 B$ ).

We observed that photoinhibition tolerance had a rather linear relationship with minimum saturating irradiance [or to a lesser degree with maximum (relative) electron transport rate]; the lower the saturating irradiance, the higher was the alga's tolerance to photoinhibition (Fig. 4). Does, therefore, the capacity for a high electron transfer rate under high light have something to do with the alga's susceptibility to PSII damage? Our result is in agreement with the suggestion that by increasing the rate of certain backward electron transfer reactions in PSII (on the expense of forward reactions), a desert alga Chlorella ohadii is able to acquire exceptional tolerance against high light, as these backward reactions may decrease singlet oxygen production by PSII (Treves et al. 2016). In addition, a high light-tolerant mutant of $C$. reinhardtii showed slower electron transfer from $\mathrm{Q}_{\mathrm{A}}$ to $\mathrm{Q}_{\mathrm{B}}$ and tolerance to PSII photoinhibition (Virtanen et al. 2019). What are the benefits of a slow forward electron transfer, one may wonder, as it could be assumed to lead to a slow growth rate? However, when light is not limiting, slow forward electron transfer capacity of PSII may not be critical; indeed, C. ohadii and the $C$. reinhardtii mutant grow and accumulate biomass efficiently under high light (Treves et al. 2016, Virtanen et al. 2019). Here, at $200 \mu \mathrm{mol}\left(\right.$ photon) $\mathrm{m}^{-2} \mathrm{~s}^{-1}$, different electron transfer capacities of the algae (Fig. 4) did not translate to different turbidostat growth rates (Table 1). We also did not observe a correlation between $\mathrm{Q}_{\mathrm{A}}^{-}$reoxidation rates (Fig. 5) and photoinhibition tolerance of the algae (Fig. 3). Still, the hypothesis that gained photoinhibition tolerance comes with the price of a slower forward electron transfer is intriguing, as it could be yet another regulatory point that maintains the cost to benefit ratio of PSII repair and electron transfer to carbon fixation optimal. Indeed, Li and Campbell (2013) showed that while growth light is increased, which increased the growth rate, Thalassiosira pseudonana became more susceptible to photoinhibition.

In the present study, the photobioreactor experiments were conducted once with each algal species. Calculations of turbidostat growth rates and error bars associated with the calculated values are based on measurement of the accumulation of effluent every $6 \mathrm{~h}$ of growth and do not represent true biological repetitions. The turbidostat growth rates were $0.68-0.94 \mathrm{~d}^{-1}$, and therefore the cell population in the reactor turned over in 1.1 to $1.5 \mathrm{~d}$.

Conclusions: We have shown that batch growth is an inadequate method to predict the growth rate during a continuous culture, due to, e.g., different light conditions. E. oleoabundans was very susceptible to photoinhibition of PSII and grew slowly under batch culture but otherwise tolerance to photoinhibition did not automatically increase the growth rate. We found a correlation between photoinhibition tolerance and fluorescence parameters indicating a slow forward electron transport of PSII. However, the observation requires further studies.

\section{References}

Barbera E., Grandi A., Borella L. et al.: Continuous cultivation as a method to assess the maximum specific growth rate of photosynthetic organisms. - Front. Bioeng. Biotechnol. 7: 274, 2019.

Barranguet C., Kromkamp J.: Estimating primary production rates from photosynthetic electron transport in estuarine microphytobenthos. - Mar. Ecol. Prog. Ser. 204: 39-52, 2000.

Borowitzka M.A., Vonshak A.: Scaling up microalgal cultures to commercial scale. - Eur. J. Phycol. 52: 407-418, 2017.

Campbell D.A., Serôdio J.: Photoinhibition of photosystem II in phytoplankton: processes and patterns. - In: Larkum A., Grossman A., Raven J. (ed.): Photosynthesis in Algae: Biochemical and Physiological Mechanisms. Advances in Photosynthesis and Respiration (Including Bioenergy and Related Processes). Vol. 45. Pp. 329-365. Springer, Cham 2020.

Eilers P.H.C., Peeters J.C.H.: A model for the relationship between light intensity and the rate of photosynthesis in phytoplankton. - Ecol. Model. 42: 199-215, 1988.

Flynn K.J.: Going for the slow burn: why should possession of a low maximum growth rate be advantageous for microalgae? Plant Ecol. Divers. 2: 179-189, 2009.

García-Cubero R., Kleinegris D.M.M., Barbosa M.J.: Predicting biomass and hydrocarbon productivities and colony size in continuous cultures of Botryococcus braunii showa. Bioresource Technol. 340: 125653, 2021.

Grobbelaar J.U., Nedbal L., Tichý V.: Influence of high frequency 
light/dark fluctuations on photosynthetic characteristics of microalgae photoacclimated to different light intensities and implications for mass algal cultivation. - J. Appl. Phycol. 8: 335-343, 1996

Hindersin S., Leupold M., Kerner M., Hanelt D.: Irradiance optimization of outdoor microalgal cultures using solar tracked photobioreactors. - Bioprocess Biosyst. Eng. 36: 345$355,2013$.

Inskeep W.P., Bloom P.R.: Extinction coefficients of chlorophyll $a$ and $b$ in N,N-dimethylformamide and $80 \%$ acetone. - Plant Physiol. 77: 483-485, 1985.

Komenda J.: Photosystem 2 photoinactivation and repair in the Scenedesmus cells treated with herbicides DCMU and BNT and exposed to high irradiance. - Photosynthetica 35: 477480, 1998.

Levasseur W., Taidi B., Lacombe R. et al.: Impact of seconds to minutes photoperiods on Chlorella vulgaris growth rate and chlorophyll $a$ and $b$ content. - Algal Res. 36: 10-16, 2018.

Li G., Campbell D.A.: Rising $\mathrm{CO}_{2}$ interacts with growth light and growth rate to alter photosystem II photoinactivation of the coastal diatom Thalassiosira pseudonana. - PLoS ONE 8: e55562, 2013.

Miyata K., Noguchi K., Terashima I.: Cost and benefit of the repair of photodamaged photosystem II in spinach leaves: roles of acclimation to growth light. - Photosynth. Res. 113: 165-180, 2012

Murata N., Nishiyama Y.: ATP is a driving force in the repair of photosystem II during photoinhibition. - Plant Cell Environ. 41: 285-299, 2018.

Murphy C.D., Roodvoets M.S., Austen E.J. et al.: Photoinactivation of Photosystem II in Prochlorococcus and Synechococcus. - PLoS ONE 12: e 0168991, 2017.

Nath K., Jajoo A., Poudyal R.S. et al.: Towards a critical understanding of the photosystem II repair mechanism and its regulation during stress conditions. - FEBS Lett. 587: 3372-3381, 2013.

Neale P.J., Melis A.: Algal photosynthetic membrane complexes and the photosynthesis-irradiance curve: a comparison of light-adaptation responses in Chlamydomonas reinhardtii (Chlorophyta). - J. Phycol. 22: 531-538, 1986.

Pätsikkä E., Kairavuo M., Šeršen F. et al.: Excess copper predisposes photosystem II to photoinhibition in vivo by outcompeting iron and causing decrease in leaf chlorophyll. Plant Physiol. 129: 1359-1367, 2002.

Raven J.A.: The cost of photoinhibition. - Physiol. Plantarum 142: 87-104, 2011.

Rippka R., Deruelles J., Waterbury J.B. et al.: Generic assignments, strain histories and properties of pure cultures of cyanobacteria. - Microbiology 111: 1-61, 1979.

Schreiber U., Klughammer C., Kolbowski J.: Assessment of wavelength-dependent parameters of photosynthetic electron transport with a new type of multi-color PAM chlorophyll fluorometer. - Photosynth. Res. 113: 127-144, 2012.

Serôdio J., Campbell D.A.: Photoinhibition in optically thick samples: effects of light attenuation on chlorophyll fluorescence-based parameters. - J. Theor. Biol. 513: 110580, 2021

Sipka G., Magyar M., Mezzetti A. et al.: Light-adapted chargeseparated state of photosystem II: Structural and functional dynamics of the closed reaction center. - Plant Cell 33: 12861302, 2021.

Stensjö K., Vavitsas K., Tyystjärvi T.: Harnessing transcription for bioproduction in cyanobacteria. - Physiol. Plantarum 162: 148-155, 2018.

Su Y., Song K., Zhang P. et al.: Progress in microalgae biofuel's commercialization. - Renew. Sust. Energ. Rev. 74: 402-411, 2017.

Treves H., Raanan H., Kedem I. et al.: The mechanisms whereby the green alga Chlorella ohadii, isolated from desert soil crust, exhibits unparalleled photodamage resistance. - New Phytol. 210: 1229-1243, 2016.

Tyystjärvi E.: Photoinhibition of photosystem II. - In: Jeon K.W. (ed.): International Review of Cell and Molecular Biology. Vol. 300. Pp. 243-303. Elsevier, Amsterdam 2013.

Valev D., Silva Santos H., Tyystjärvi E.: Stable wastewater treatment with Neochloris oleoabundans in a tubular photobioreactor. - J. Appl. Phycol. 32: 399-410, 2020.

Virtanen O., Khorobrykh S., Tyystjärvi E.: Acclimation of Chlamydomonas reinhardtii to extremely strong light. Photosynth. Res. 147: 91-106, 2021.

Virtanen O., Valev D., Kruse O. et al.: Photoinhibition and continuous growth of the wild-type and a high-light tolerant strain of Chlamydomonas reinhardtii. - Photosynthetica 57: 617-626, 2019.

(C) The authors. This is an open access article distributed under the terms of the Creative Commons BY-NC-ND Licence. 